\title{
Energy-Dependent Disassembly of Self-Assembled SNARE Complex: Observation at Nanometer Resolution Using Atomic Force Microscopy
}

Aleksandar Jeremic ${ }^{\dagger}$, Anthony S. Quinn ${ }^{\dagger \dagger}$, Won Jin $\mathrm{Cho}^{\dagger}$, Douglas J. Taatjes ${ }^{\dagger \dagger}$ and Bhanu P. Jena ${ }^{\dagger, ~ * ~}$

Wayne State University School of Medicine, Department of Physiology, Detroit, MI, 48201

University of Vermont, Department of Pathology, Burlington, VT, 05405

\section{Supporting Information}

Preparation of Proteoliposomes All lipids were obtained from Avanti Polar Lipids (Alabaster, AL). A $5 \mathrm{mM}$ lipid stock solution was prepared by mixing lipid solution in chloroform-DOPC (1,2-dioleoyl phosphatidylcholine): DOPS (1,2-dioleoyl phosphatidylserine) in 70:30 mol/mol ratios in glass test tubes. The lipid mixture was dried under gentle stream of nitrogen and resuspended in decane. Lipids were suspended in buffer containing $10 \mathrm{mM}$ Hepes-NaOH $[\mathrm{pH}$ $=7.5 \mathrm{~J}$ and $140 \mathrm{mM} \mathrm{NaCl}$ by vortexing for $5 \mathrm{~min}$ at room temperature. Unilamellar vesicles were formed following sonication for $2 \mathrm{~min}$. Typically, vesicles ranging in size from $0.2-2 \mu \mathrm{m}$ in diameter were obtained as assessed by atomic force microscopy (AFM). Two sets of proteoliposomes were prepared by gently mixing either t-SNARE complex (Syntaxin-1/SNAP25; final concentration $5 \mu \mathrm{g} / \mathrm{ml}$ ) or VAMP2-His ${ }_{6}$ (final concentration $2.5 \mu \mathrm{g} / \mathrm{ml}$ ) with liposomes (Cho et al., 2002; Jeremic et al., 2004), followed by three freeze/thaw cycles to enhance protein reconstitution at the vesicles membrane.

Light Scattering Measurements Kinetics of association and dissociation of t-SNARE and vSNARE reconstituted vesicles in solution were monitored by right angle light scattering assay with excitation and emission wavelength set at $600 \mathrm{~nm}$ in a Hitachi F-2000 spectrophotometer (Wilschut et al., 1981; Jeremic et al., 2004). Equal volume $(7 \mu 1)$ of t-SNARE and v-SNARE reconstituted vesicle suspension was injected into the cuvette containing $700 \mu \mathrm{l}$ of assay buffer $\left(140 \mathrm{mM} \mathrm{NaCl}, 10 \mathrm{mM}\right.$ Hepes $\left.\mathrm{pH}=7.4,2 \mathrm{mM} \mathrm{CaCl}_{2}\right)$ at a final lipid concentration of $100 \mu \mathrm{M}$ at $37^{\circ} \mathrm{C}$. NSF $(1 \mu \mathrm{g} / \mathrm{ml})$ was injected in to reaction mixture following addition of proteoliposomes containing ATP-Mg $(50 \mu \mathrm{M})$ or its non-hydrolyzable analog AMP-PNP $(50 \mu \mathrm{M})$. Changes in the light scattering were continuously monitored for a $5 \mathrm{~min}$ period. Values are expressed as intensities of scattered light (arbitrary units) taken at $1 \mathrm{~min}$ after addition of proteoliposomes or $\mathrm{NSF}$, after which interactions between vesicles in solution reached a steady state. Student's $t$-test was used for comparisons between groups with significance established at $\mathrm{p}<0.05$ (*). 
Immunoblot Analysis of SNARE complex disassembly Quantitative assessment of SNARE complex disassembly was determined using immunochemical analysis. As in the light scattering studies, t-SNARE and v-SNARE reconstituted vesicles were incubated together for 5 min at $37^{\circ}$ $\mathrm{C}$ in the presense or absence of NSF, ATP and/or AMP-PNP. Interactions were stopped by addition of Laemmli reducing sample preparation buffer at R.T. and the SNARE complex formed, were resolved in a $12.5 \%$ SDS-PAGE. Proteins were electrotransferred to nitrocellulose sheets for immunoblot analysis using syntaxin 1 specific antibody (1:2000) (Alomone lab, Israel). Immunobands were visualized using a chemiluminescence detection system (Amersham Biosciences, UK) and photographed using a Kodak Image Station 440. Densytometric analysis of the immunobands were performed using the Kodak 1D Image Analysis software and is presented as relative intensities or optical density (O.D.). The approximately $70 \mathrm{kDa}$ band is the $\mathrm{t}$-/v-SNARE complex, and the lower $33 \mathrm{kDa}$ band that of Syntaxin-1. Student's $t$-test was used for comparisons between groups with significance established at $p<0.05(*)$ and $p<0.01(* *)$.

Atomic force microscopy Atomic force microscopy was performed on mica and on lipid membrane in buffer. Lipid membrane alone or in the presence of t-SNAREs, followed by $v$ SNARE or v-SNARE reconstituted vesicles, NSF and ATP, were imaged using the Nanoscope IIIa AFM from Digital Instruments. (Santa Barbara, CA). Following addition of t-SNAREs, vSNARE, or NSF, the sample was washed 10 times using the sample buffer, prior to imaging using the AFM. Images were obtained both in the "contact" and "tapping" mode in fluid of the same area. However, all images presented in this manuscript were obtained in the "tapping" mode in fluid, using silicon nitride tips with a spring constant of $0.38 \mathrm{~N} . \mathrm{m}^{-1}$, and an imaging force of $<200 \mathrm{pN}$. Images were obtained at line frequencies of $2 \mathrm{~Hz}$, with 512 lines per image, and constant image gains. Topographical dimensions of SNARE complexes and lipid vesicles were analyzed using the software nanoscope IIIa4.43r8, supplied by Digital Instruments.

Section analysis of the t-SNARE complexes in a lipid membrane, before and after addition of V-SNARE, demonstrates moderate changes in both shape and size of the complexes. A $5 \%$ increase in diameter and $40 \%$ increase in height have previously been demonstrated following addition of v-SNARE to t-SNARE complexes in lipid membrane (Cho et al., 2002 and Fig 3B in article). In contrast to the SNARE complex formed when t/v-SNAREs were added to the same bilayer, t-SNAREs and v-SNARE in opposing bilayers interact and arrange in specific ring pattern, forming pore-like structures (Cho et al., 2002 , Cho et al., 2005 and manuscript summary).

The time course of SNARE complex disassembly is clearly provided in the light scattering experiments. The AFM micrographs further confirm pictorially, the NSF-ATPinduced t-/v-SNARE disassembly, at $\mathrm{nm}$ resolution. 
All AFM images of SNARE complexes formed under different conditions are highly reproducible. Continued imaging of these complexes under different forces, do not alter them. However, only in the presence of NSF-ATP, do t-/v-SNAREs disassemble, as demonstrated in the AFM micrographs (Fig. 1 and 2). Additionally, the SNARE complex disassembly observed by AFM, is a further conformation of both the light scattering and immunochemical studies.

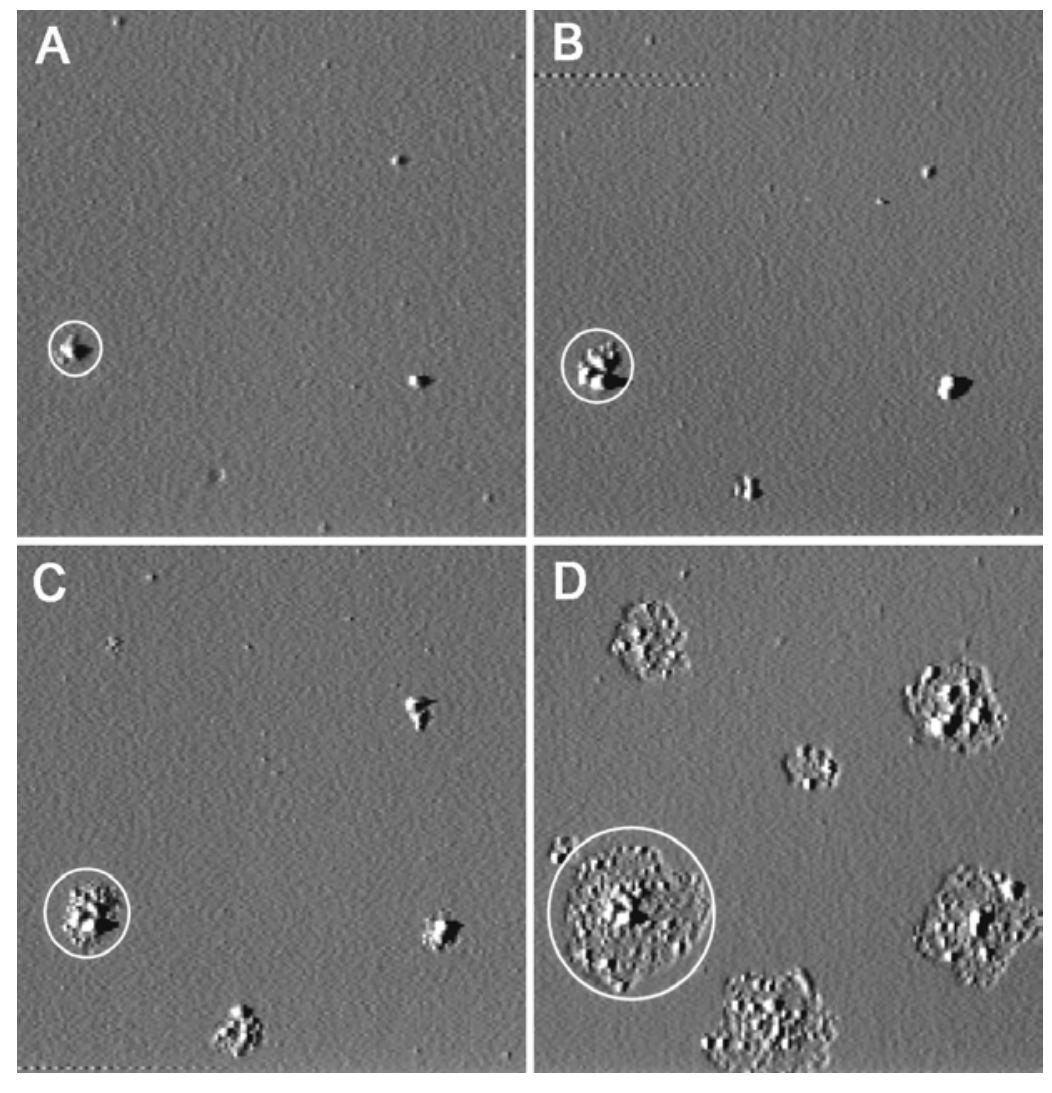

Fig1. High resolution AFM micrographs of the formation and NSF-ATP induced disassembly of several t-/v-SNARE complexes, within an experiment. AFM micrograph of t-SNARE on lipid membrane (A), followed by addition of v-SNARE (B), then NSF (C), and ATP (D). Note the disassembly of the t-/v-SNARE complex in D. 


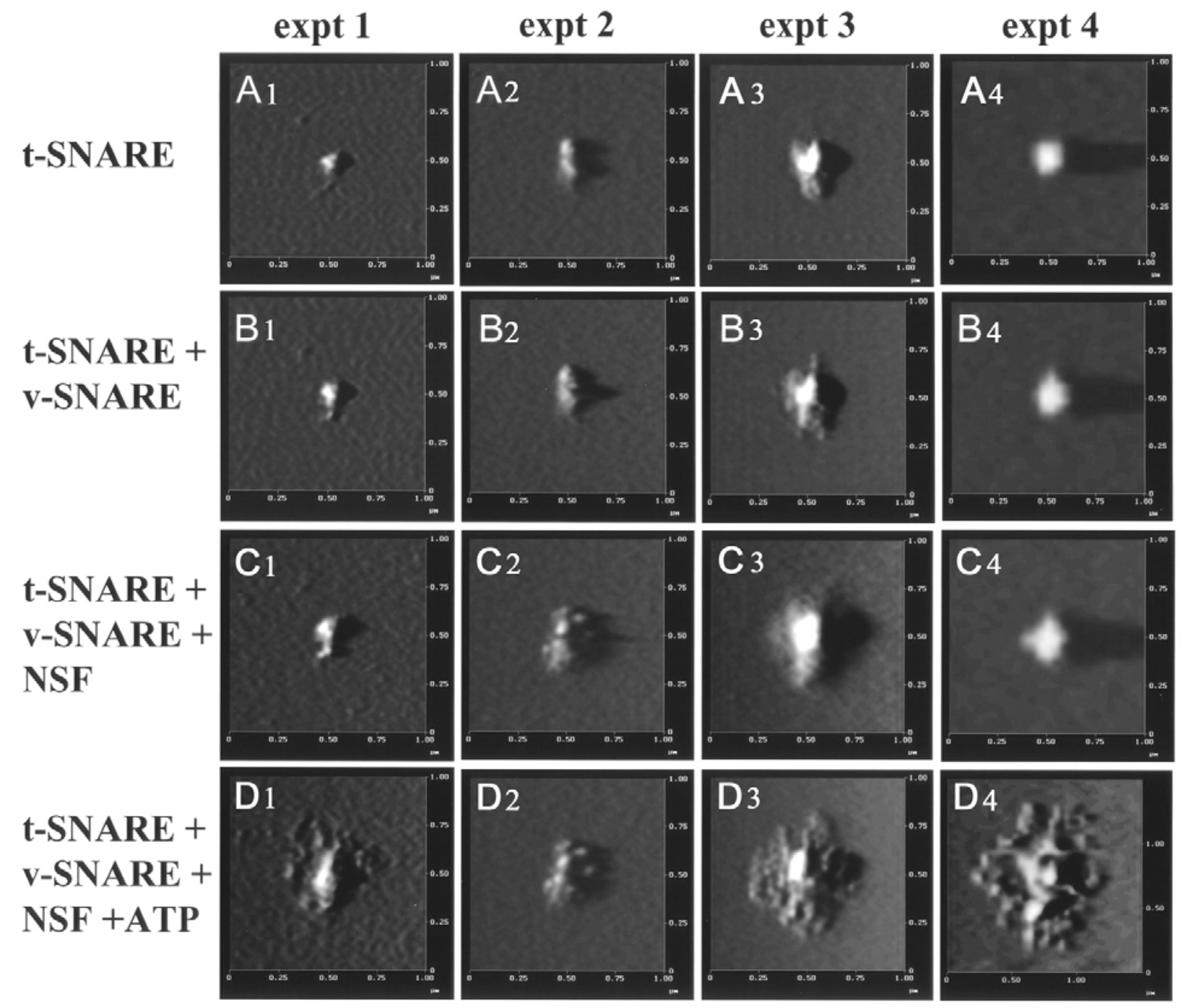

Fig 2. High resolution AFM micrographs of the formation and NSF-ATP induced disassembly of $t-/ v-S N A R E$ complexes in four separate experiments.

\section{REFERENCES}

Cho SJ, Kelly M, Rognlien KT, Cho J, Hoerber JKH, Jena BP. (2002) SNAREs in opposing bilayers interact in a circular array to form conducting pores. Biophys $J$ 83:2522-2527.

Cho WJ, Jeremic A, Jena BP (2005) Size of supramolecular SNARE complex: Membranedirected self-assembly. J. Am. Chem. Soc. 127, 10156-10157.

Jeremic A, Kelly M, Cho WJ, Cho SJ, Horber JKH, Jena BP. (2004) Calcium drives fusion of SNARE-apposed bilayers. Cell Biol Int 28:19-31. 
Wilschut, J., Duzgunes, N., and Papahadjopoulos, D. (1981) Calcium/magnesium specificity in membrane fusion: kinetics of aggregation and fusion of phosphatidylserine vesicles and the role of bilayers curvature. Biochemistry 20:3126-3133. 